\title{
Carpal tunnel syndrome associated with erythema multiforme during pregnancy
}

\author{
Astrit M. Gashi', Jakup Ismajli', Gent Sopa' ${ }^{1}$ Genc Mekaj², Albulena Gashi² \\ ${ }^{1}$ Department of Obstetrics and Gynecology, \\ University Clinical Center of Kosovo, University of Pristine, Kosovo \\ ${ }^{2}$ University of Pristine, Kosovo
}

\begin{abstract}
Carpal tunnel syndrome is a peripheral mononeuropathy that is caused by median nerve compression inside the carpal canal. The primary cause of carpal tunnel syndrome is the median nerve compression inside the carpal canal. This compression is caused by an increase in internal carpal canal pressure. The carpal tunnel has a fixed capacity; therefore, each condition that provokes a constriction of the inside of the canal will directly increase the internal pressure and consequently compress the median nerve. Most of the causes of carpal tunnel syndrome are idiopathic. Secondary causes of carpal tunnel syndrome include various pathologies the following: space-occupying lesions (tumors, hypertrophic synovial tissue, fracture callus, and osteophytes); metabolic and physiological (pregnancy, hypothyroidism, and rheumatoid arthritis); infections; neuropathies (associated with diabetes mellitus or alcoholism), etc. During pregnancy, weight gain and swelling in the hands can cause carpal tunnel syndrome, which comes as a result of decompression of the median nerve. The increase in body fluids during pregnancy is responsible for the largest portion of weight gain and physiologic edema. Fluid accumulates during pregnancy due to hormonal changes that occur. The incidence of neurophysiologically confirmed pregnancy-related carpal tunnel syndrome ranged from $7 \%$ to $43 \%$, whereas the incidence of clinically diagnosed pregnancy-related carpal tunnel syndrome ranged from $31 \%$ to $62 \%$. In general, carpal tunnel syndrome is presented with symptoms such as pain, numbness or paresthesias, tingling along the branches of the median nerve in the hand and wrist. The diagnosis of carpal tunnel syndrome is decided based on symptoms, clinical signs, and electrophysiological study results. Occasionally, the carpal tunnel syndrome is associated with other pathologies. At our clinic, a case appeared with carpal tunnel syndrome, in which the skin manifestations predominated over the neurologic symptoms. The skin changes included erythema multiforme. Erythema multiforme is a skin condition considered to be a hypersensitivity reaction to infections or drugs. These findings suggest that carpal tunnel syndrome and erythema multiforme coexist in this case. This is unprecedented in the literature. This co-existence of these diseases had no direct fetal impact, but influences the quality of life of the pregnant mother (with predominance the psychic aspect).
\end{abstract}

Keywords: pregnancy, carpal tunnel syndrome, erythema multiforme, rarely

\section{INTRODUCTION}

The carpal tunnel is an inelastic fibro-osseous, tunnel defined by the carpal bones and the flexor retinaculum. The medial border of the carpal tunnel consists of the pisiform bone and the hamate bone or unciform bone, while the lateral border consists of the scaphoid bone and the trapezoid bone. On the palmar surface, they are covered by the flexor retinaculum (transverse carpal ligament, or anterior annular ligament). Longitudinally the carpal tunnel is $2-2.5 \mathrm{~cm}$, and with a mean width of $20 \mathrm{~mm}$, expanding to a mean width of $25 \mathrm{~mm}$ at its proximal and distal margins.

The median nerve and flexor tendon passes through this carpal tunnel. The median nerve is a mixed sensory and motor nerve (is composed of branches from the $\mathrm{C} 5$ through $\mathrm{T} 1$ spinal cord nerve roots).

Carpal tunnel syndrome is a peripheral mononeuropathy that is caused by median nerve compression inside the carpal canal. The primary cause of carpal tunnel syndrome is the median nerve 
compression inside the carpal canal. This compression is caused by an increase in internal carpal canal pressure. If the wrist is in flexion or extreme extension, the internal space of the carpal tunnel is reduced. The carpal tunnel has a fixed capacity; therefore, each condition that provokes a constriction of the inside of the canal will directly increase the internal pressure and consequently compress the median nerve. For example, any content such as; edema, inflammation, hemorrhage, deposits of pathologic substances such as calcium uric, within the canal can decrease the available carpal canal space, causing compression of its internal structures. Most of the causes of carpal tunnel syndrome are idiopathic.

Secondary causes of carpal tunnel syndrome include various pathologies the following: space-occupying lesions (tumors, hypertrophic synovial tissue, fracture callus, and osteophytes); metabolic and physiological (pregnancy, hypothyroidism, and rheumatoid arthritis); infections; neuropathies (associated with diabetes mellitus or alcoholism); and familial disorders (collagen diseases, and acromegaly) etc.

Studies show that risk factors in the general population included repetitive activities requiring wrist extension or flexion, obesity, very rapid dieting, shorter height, hysterectomy without oophorectomy, and menopause. Occasionally, the carpal tunnel syndrome is associated with other pathologies such as De Quervain syndrome, Motta disease, Raynaud phenomenon, and epicondylitis. Various causes of carpal tunnel syndrome are described in the medical literature. Only a part of them are known.

We present a rare case; a pregnant woman with carpal tunnel syndrome associated with erythema multiforme. The increase in body fluids during pregnancy is responsible for the largest portion of weight gain and physiologic edema. Fluid accumulates during pregnancy because the adrenal glands produce more of the hormones that make the body retain fluids (aldosterone and cortisol). Less commonly, edema during pregnancy can also result from other disorders such as preeclampsia, deep vein thrombosis, diabetes mellitus, cellulitis, etc. Fluids may accumulate, causing swelling in the face, hands, or feet and weight gain. During pregnancy, weight gain and swelling in the hands can cause carpal tunnel syndrome, which comes as a result of decompression of the median nerve. J.Th. Wallace and A.W. Cook (1957); reported for the first time the pregnancy-related carpal tunnel syndrome (1). One systematic review found that, the incidence of neurophysiologically confirmed pregnancy-related carpal tunnel syndrome ranged from $7 \%$ to $43 \%$, whereas the incidence of clinically diagnosed pregnancy-related carpal tunnel syndrome ranged from $31 \%$ to $62 \%(2,3)$.

In general, carpal tunnel syndrome is presented with symptoms such as pain, numbness or paresthesias, tingling along the branches of the median nerve in the hand and wrist. Especially, in the first three fingers and the radial side of the fourth finger $(4,5)$. Overall, the severity of symptoms increased with increasing gestational weeks (mostly during the last trimester). The diagnosis of carpal tunnel syndrome is decided on the basis of symptoms, clinical signs, and electrophysiological study results. During clinical examination, Tinel's sign and Phalen's sign are two clinical tests that can be performed to assess whether an individual has carpal tunnel syndrome or not. The final diagnosis is decided with the help of electrodiagnostic testing (electromyography), but very often this test is unnecessary.

Treatment of carpal tunnel syndrome consists of conservative treatments (including; wrist splint, nonsteroidal anti-inflammatory drugs, corticosteroids) and surgical treatments (endoscopic surgery or open surgery). The goal of carpal tunnel surgery is to relieve pressure by cutting the ligament pressing on the median nerve.

At our clinic, a case appeared with carpal tunnel syndrome, in which the skin manifestations predominated over the neurologic symptoms. The skin changes included erythema multiforme. Erythema multiforme is a skin condition considered to be a hypersensitivity reaction to infections or drugs. These findings suggest that carpal tunnel syndrome and erythema multiforme coexist in this case. This is unprecedented in the literature.

\section{CASE PRESENTATION}

A 34-year-old woman was referred to the Obstetrics and Gynecology Clinic because of pain, numbness, tingling in her hands, but also because 
of cutaneous manifestations in the form of lesions or pink macules. The lesions had a regular round shape and three concentric zones: a central darker area, a pink or edematous zone, and a peripheral red ring. The lesions were located on the dorsal surfaces of the hands. The patient declares that cutaneous manifestations were presented seven days ago. Initially, with itching and burning at the site of the eruption later developed macules that then became papules or plaques. Meanwhile, symptoms such as pain, numbness, tingling in her hands were presented to her eight weeks ago. Initially, with mild intensity, but day after day they were aggravated. Due to pain and numbness in her hands, she had used, without a prescription of non-steroidal anti-inflammatory drugs (concretely, nimesulide granules for oral suspension). Three days after the use of non-steroidal anti-inflammatory drugs (NSAIDs), changes in the skin appear. These skin manifestations predominated over neurologic symptoms. She is a primipara and was admitted to the Clinic in week 39 of gestation. She did not have diabetes mellitus, rheumatoid arthritis, hypothyroidism, collagen diseases, hypertension, or any other disease. She had a height of $163 \mathrm{~cm}$, and weight was $74 \mathrm{~kg}$. The body mass index (BMI) was 27.9. Blood pressure was 120/75 mm Hg. On vaginal examination: vulva without any macroscopically visible change. Vagina in width and normal length, and had a slight discharge of secretions. The cervix: shortened by 50\%, 1.5-centimeter dilated, intact amniotic membrane, present head, heartbeat is clear, rhythmical, $135 \mathrm{bpm}$. The uterus is normotonus. The complete blood count $(\mathrm{CBC})$ was in the referent values. There was no proteinuria. On ultrasonographic examination: fetal biometrics corresponds to the 39th week of pregnancy and fetal heart rate (FHR) was $144 \mathrm{bpm}$. Her main complaints were pain, numbness, tingling along the branches of the median nerve in her hand and wrist. Especially: in the first three fingers and the radial side of the fourth finger (see figure1, below). These clinical signs appeared in the last trimester of pregnancy and were of mild intensity. The electromyographic study was consistent with bilateral carpal tunnel syndrome (CTS). No abnormal results were found in biochemical, hematological, or immunological determinations.

But, the patient also had been some skin changes that were atypical in this case. Skin changes in- cluded: an urticarial plaque that constantly expands and in the center was a small papule or vesicle, where it emptied, flattens, and then may clear. An intermediate ring develops and becomes raised, pale, and edematous. On the periphery of the urticarial plaque, developed concentric rings (see figure 2 , below). The patient states that the changes in the skin started 7 days ago, with a purpuric macula, which was constantly expanding, and accompanied by pain and pruritus. These changes were presented in both hands.

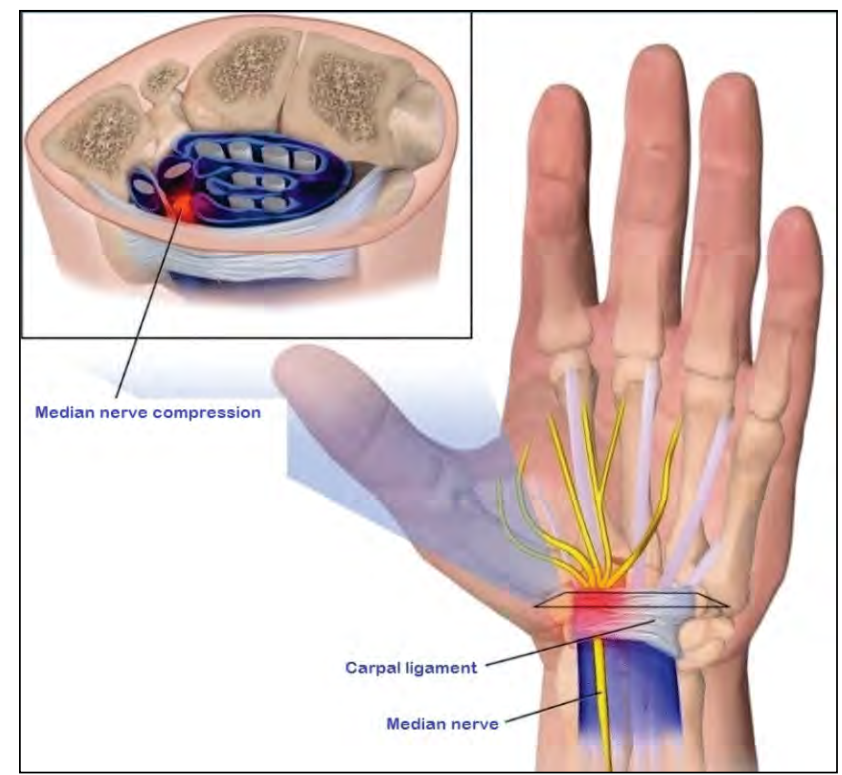

FIGURE 1. Carpal tunnel syndrome (illustration, obtained with permission)

A consultation was done with a dermatologist, who after clinical examinations diagnosed the case as erythema multiforme. These findings suggest that carpal tunnel syndrome and erythema multiforme coexist in this case. This is unprecedented in the literature. So that was our reason for reporting it.

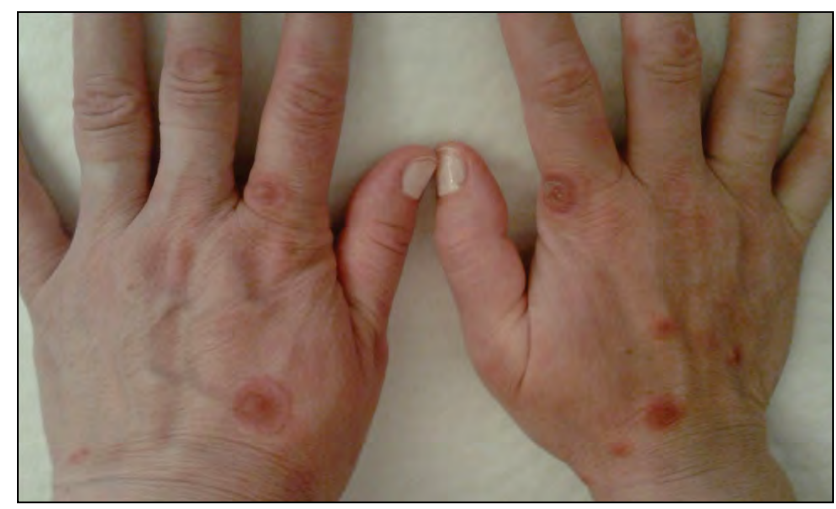

FIGURE 2. Erythema multiforme in both hands 


\section{DISCUSSION}

During pregnancy, many physiological changes occur in the body due to the effect of hormones. These physiological changes occur in all systems of the organism, to pregnant women, in the reproductive system, cardiovascular system, endocrine system, respiratory system, gastrointestinal system, and urinary system, changes in the breasts, the skin, and changes in body weight. These changes are considered normal, but can sometimes promote the development of various pathologies.

In our case, carpal tunnel syndrome during pregnancy is believed to be caused by local edema in the carpal tunnel due to hormonal changes that favor fluid retention in the body. Other risk factors found in our case were generalized edema in the body, obesity, shorter height. Was not found any other causes such as; tumors, hypertrophic synovial tissue, fracture callus, osteophytes, hypothyroidism, rheumatoid arthritis, diabetes mellitus, or alcoholism. In many cases, the etiology of carpal tunnel syndrome is idiopathic.

These patients have typical signs and symptoms of carpal tunnel syndrome, but no significant past medical history (no history of trauma, diabetes mellitus, crystalline arthropathy, metabolic disease, endocrine imbalance, inflammatory arthritis, and other related systemic diseases), documented abnormalities in preoperative electrodiagnostic studies, with no evident structural cause of compression of the median nerve intraoperatively.

Medical literature reports on various studies in which the incidence of carpal tunnel syndrome has various causes. Carpal tunnel syndrome often occurs during pregnancy and mostly appears in the third trimester (6-8). The prevalence rates of carpal tunnel syndrome during pregnancy vary between $<1$ up to 62 percent $(9,11)$. Carpal tunnel syndrome during pregnancy is believed to be caused by local edema in the carpal tunnel due to hormonal changes $(10,11)$.

In our case, the diagnosis of carpal tunnel syndrome was made in the 39th week of pregnancy. The patient had mild to moderate symptoms at the beginning of the third trimester, and the severity of the symptoms increased near the term of birth.

Carpal tunnel syndrome can be isolated or associated with other pathologies such as De Quervain syndrome, Motta disease, Raynaud phenomenon, epicondylitis, or shoulder pathologies (12-14). Other pathologies that cause synovial inflammation and edema can cause compression of the median nerve and the onset of carpal tunnel syndrome, for example, scleroderma, lupus, etc. In 3-6\% of patients afflicted with Sjögren syndrome, there is an associated carpal tunnel syndrome (15). A study reported that five cases of carpal tunnel syndrome were presented (or associated) with cutaneous alterations of the connective tissue such as fasciitis, discoid lupus, panniculus lupus (16). Other cases that have been reported to have an association with carpal tunnel syndrome are dermatomyositis, polymyositis, and lymphoma etc. (17).

In our case, the manifestation of carpal tunnel syndrome symptoms was also associated with skin changes that respond to erythema multiforme. The peculiarity of this case was that carpal tunnel syndrome and erythema multiforme coexist. This coexistence of carpal tunnel syndrome and erythema multiforme has not been described in the literature. Erythema multiforme is a skin condition considered to be a hypersensitivity reaction to infections or drugs (18). Cutaneous manifestations in erythema multiforme commonly include lesions such as sharply red or pink macules that then become papular. The papules may enlarge gradually into plaques several centimeters in diameter. The central portion of the papules or plaques gradually becomes darker red or purpuric. Crusting occurs in the center of the papules or plaques. These lesions may be associated with itching and burning at the site of the eruption. The lesions have a regular round shape and three concentric zones: a central dusky or darker red area, a paler pink or edematous zone, and a peripheral red ring. These lesions usually appear symmetrically in both extremities, but also the body. Lesions on the dorsal surfaces of the hands and extensor aspects of the extremities are most characteristic (18-21).

Due to the mild or moderate symptomatology of carpal tunnel syndrome, the patient was self-treated with non-steroidal anti-inflammatory drugs (NSAIDs). She states that 3 days after self-treatment for pain, changes in the skin had begun to appear. After 7 days, she was referred to our clinic. Dermatological symptomatology predominated over neurological symptomatology. Neurologists, 
dermatologists, and orthopedics were consulted, diagnoses are made and adequate therapies are prescribed.

In the 40th week of pregnancy due to obstetric indications, the patient performs the birth by Cesarean section and gives birth to a male baby with a bodyweight of 4,070 grams, length $54 \mathrm{~cm}$, and Apgar score 8/9. Signs and symptoms of carpal tunnel syndrome disappeared completely sixth weeks after birth. Within eight months after birth, the signs of erythema multiforme were repeated twice.

\section{CONCLUSIONS}

From numerous studies, it is known that pregnancy is a possible cause of carpal tunnel syndrome, this happens because of hormonal changes that occur during pregnancy affecting fluid retention (physiological edema). This edema of the tissues around the median nerve can decrease the available carpal canal space, causing compression of its internal structures (specifically compression of the median nerve). It is known that the carpal tunnel has a fixed capacity; therefore, each condition that provokes a constriction of the inside of the canal will directly increase the internal pressure and consequently compress the median nerve.

During pregnancy, the signs and symptoms of carpal tunnel syndrome are mild to moderate. Mostly, the treatment is conservative and rarely requires surgical intervention. No confirmed cause was found that could be related to the occurrence of erythema multiforme. Initially, as a cause of multiform erythema was suspected in the use of non-steroidal anti-inflammatory drugs (NSAIDs). But the recurrence of signs and symptoms of erythema multiforme, even for 2 times along during the 8 months after birth, of questioned this cause.

The manifestation of carpal tunnel syndrome associated with multiform erythema during pregnancy was a case which was not described in the literature. This co-existence of these diseases had no direct fetal impact, but influences the quality of life of the pregnant mother (with predominance the psychic aspect).

\section{Informed consent}

The patient has provided informed consent for publication of the case.

Conflict of interest: none declared Financial support: none declared

\section{REFERENCES}

1. Wallace JT, Cook AW. Carpal tunnel syndrome in pregnancy: A report of two cases. American Journal of Obstetrics \& Gynecology. 1957;73(6):1333-6.

2. Padua L, Pasquale AD, Pazzaglia C, Liotta GA, Librante A, Mondelli M. Systematic review of pregnancy-related carpal tunnel syndrome. Muscle \& Nerve. 2010;42(5):697-702.

3. Meems M, Truijens SE, Spek V, Visser LH, Pop VJ. Prevalence, course and determinants of carpal tunnel syndrome symptoms during pregnancy: A prospective study. BJOG: An International Journal of Obstetrics \& Gynaecology. 2015;122(8):1112-8.

4. Dawson DM. Entrapment neuropathies of the upper extremities. New England Journal of Medicine. 1993;329(27):2013-8.

5. Ibrahim I, Khan WS, Goddard N, Smitham P. Suppl 1 - Carpal tunnel syndrome: A review of the recent literature. The Open Orthopaedics Journal. 2012; 6:69.

6. Finsen $\mathrm{V}$, Zeitlmann $\mathrm{H}$. Carpal tunnel syndrome during pregnancy Scandinavian Journal of Plastic and Reconstructive Surgery and Hand Surgery. 2006;40(1):41-5.

7. Ablove RH, Ablove TS. Prevalence of carpal tunnel syndrome in pregnant women. Wisconsin Medical Journal (WMJ). 2009;108(4):194.

8. Stolp-Smith KA, Pascoe MK, Ogburn Jr PL. Carpal tunnel syndrome in pregnancy: frequency, severity, and prognosis. Archives of Physical Medicine and Rehabilitation. 1998;79(10):1285-7.

9. Mondelli M, Rossi S, Monti E, Aprile I, Caliandro P, Pazzaglia C, Romano C, Padua L. Prospective study of positive factors for improvement of carpal tunnel syndrome in pregnant women. Muscle \& Nerve: Official Journal of the American Association of Electrodiagnostic Medicine. 2007; 36(6):778-83.

10. Osterman M, llyas AM, Matzon JL. Carpal tunnel syndrome in pregnancy. Orthopedic Clinics. 2012;43(4):515-20.

11. Padua L, Aprile I, Caliandro P, Carboni T, Meloni A, Massi S, Mazza O, Mondelli M, Morini A, Murasecco D, Romano M. Symptoms and neurophysiological picture of carpal tunnel syndrome in pregnancy. Clinical Neurophysiology. 2001;112(10):1946-51.

12. Phalen GS. The Carpal-Tunnel Syndrome: Seventeen years'experience in diagnosis and treatment of 654 hands. JBJS. 1966;48(2):211-28.

13. Cseuz KA, Thomas JE, Lambert EH, Love JG, Lipscomb PR. Long-term results of operation for carpal tunnel syndrome. Journal of Occupational and Environmental Medicine. 1966;8(10):560.

14. Hybbinette $\mathrm{CH}$, Mannerfelt $\mathrm{L}$. The Carpal Tunnel Syndrome: $\mathrm{A}$ Retrospective Study of IOQ Operated Patients. Acta Orthopaedica Scandinavica. 1975;46(4):610-20.

15. Binder A, Snaith ML, Isenberg D. Sjögren's syndrome: A study of its neurological complications. Rheumatology. 1988;27(4):275-80.

16. Winkelmann RK, Connolly SM, Doyle JA. Carpal tunnel syndrome in cutaneous connective tissue disease: Generalized morphea, lichen sclerosus, fasciitis, discoid lupus erythematosus, and lupus panniculitis. Journal of the American Academy of Dermatology. 1982;7(1):94-9.

17. Quinones CA, Perry HO, Rushton JG. Carpal tunnel syndrome in dermatomyositis and scleroderma. Archives of Dermatology. 1966;94(1):20-5

18. Lamoreux MR, Sternbach MR, Hsu WT. Erythema multiforme. American Family Physician. 2006;74(11):1883-8.

19. Trayes KP, Love G, Studdiford JS. Erythema multiforme: Recognition and management. American Family Physician. 2019;100(2):82-8.

20. Huff JC. Erythema maltiforme and latent herpes simplex infection. Semim Dermatol. 1992;11:207.

21. Shin HT, Chang MW. Drug eruptions in children. Current Problems in Pediatrics. 2001;31(7):207-34. 